04;09

\title{
Экранирование потока испаренного материала слоем Ленгмюра, формирующимся при контакте горячей плазмы с металлом
}

\author{
(C) В.М. Кожевин, М.В. Горохов А.А. Борматов \\ Физико-технический институт им. А.Ф. Иофффе РАН, Санкт-Петербург \\ E-mail: vmk@pltec.ioffe.ru
}

Поступило в Редакцию 21 января 2017 г.

Проводится анализ условий, при которых эффект экранирования потока испаренных атомов слоем Ленгмюра, обусловленный ионизацией атомов в этом слое, может существенно повлиять на динамику плазмы, контактирующей с поверхностью. Показано, что учет эффекта экранирования потока испаренных атомов слоем Ленгмюра необходим во многих случаях, в частности при моделировании процессов динамики лазерного факела.

DOI: 10.21883/PJTF.2017.14.44818.16711

Эрозия материалов под воздействием горячей плазмы на поверхность материальных стенок является одной из ключевых проблем, возникающих при конструировании установок для многочисленных приложений, в частности установок термоядерного синтеза [1], а также в лазерных технологиях нанесения тонкопленочных покрытий из различных материалов [2]. Соответственно, как при конструировании таких установок, так и при выборе режимов их эксплуатации, важно иметь возможности проведения корректных оценок, позволяющих определить скорость деградации облучаемых материалов, определяемую испарением материала, а также выбросом микрокапель и кластеров с их поверхности.

В данной работе проводится анализ влияния слоя Ленгмюра, формирующегося в зоне контакта горячей плазмы и стенки, на процессы инжекции испаренного вещества в плазму. Отметим, что в моделях, используемых в настоящее время для анализа этих процессов, акцент делается на подробном описании динамики плазмы [1], при этом факт 


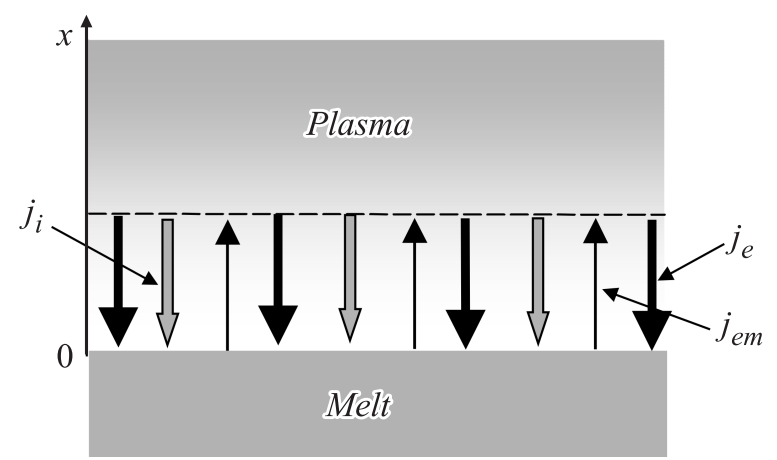

Рис. 1. Потоки электронов и ионов через слой Ленгмюра.

наличия слоя Ленгмюра нигде не учитывается и не оговаривается правомерность использования такого допущения. Скорее всего, это связано с тем, что для горячей плазмы толщина слоя Ленгмюра составляет всего лишь несколько нанометров, что много меньше длины свободного пробега ионов и атомов, обусловленной ион-нейтральными столкновениями. Соответственно, предполагается, что слой Ленгмюра можно считать прозрачным для потоков атомов, ионов и энергии. Однако этот вывод становится неоднозначным, если принять во внимание, что, как будет показано ниже, вероятность ионизации испаренных атомов в слое Ленгмюра может оказаться достаточно большой.

Важность учета эффектов ионизации связана прежде всего с тем, что ионы, возникшие в слое Ленгмюра, начинают ускоряться под воздействием сильного электрического поля в направлении, обратном скорости их первоначального движения, и могут возвращаться на стенку. Таким образом, возникает эффект экранирования плазмы от потока испаренных атомов слоем Ленгмюра. Ниже приводится описание аналитической модели, позволяющей оценить долю испаренных атомов, возвращающихся на стенку, и анализируются условия, при которых учет эффекта экранирования потока испаренных атомов становится важным.

Отметим, что ионизация атомов в слое Ленгмюра может осуществляться как вследствие столкновений электронов, пересекающих слой, с испаренными атомами, так и в процессе фотоионизации этих атомов, инициированной интенсивными радиационными потоками из плазмы.

Письма в ЖТФ, 2017, том 43, вып. 14 
Однако в данной работе мы ограничимся рассмотрением ионизации электронным ударом и не будем учитывать эффекты, обусловленные фотоионизацией атомов. Отметим, что учет фотоионизации приводит к повышению частоты ионизации атомов в слое Ленгмюра и усилению эффекта экранирования. При рассмотрении процесса ионизации атомов, обусловленной их столкновением с электронами, необходимо учитывать два типа противоположно направленных электронных потоков, пересекающих слой Ленгмюра (рис. 1), а именно, поток электронов, эмитируемый из плазмы, и поток электронов, эмитируемый с поверхности проводящей стенки. Плотность электронного тока, эмитируемого из плазмы в слой Ленгмюра, равна [3]

$$
j_{e}=e n_{p l}\left(\frac{k T_{e}}{2 \pi m_{e}}\right)^{1 / 2} \exp \left(-\frac{e U}{k T_{e}}\right)
$$

где $T_{e}$ и $n_{p l}$ - температура электронов и плотность плазмы, $e$ и $m_{e}$ - заряд и масса электрона, $U$ - падение потенциала на слое. Ток термоэлектронной эмиссии с поверхности проводящей стенки описывается формулой Ричардсона-Дэшмана с поправкой Шоттки к работе выхода $[4,5]$

$$
j_{e m}=A_{R} T^{2} \exp \left(-\frac{w-\Delta w}{k T}\right),
$$

где $A_{R}$ - постоянная Ричардсона, $T$ и $w$ - температура и работа выхода расплава, $\Delta w=e \sqrt{e E / 4 \pi \varepsilon_{0}}$ - поправка Шоттки к работе выхода, $E$ - напряженность электрического поля на поверхности материальной стенки, $\varepsilon_{0}$ - электрическая постоянная.

Использование формул (1) и (2) позволяет вычислить частоту ионизации атомов в слое Ленгмюра из равенства $e \nu_{i}=\sigma_{e} j_{e}+\sigma_{e m} j_{e m}$, где $\sigma_{e}$ и $\sigma_{e m}-$ сечения ионизации атомов электронами, эмитируемыми из плазмы с поверхности проводящей стенки соответственно. В общем случае следует учитывать зависимость сечений ионизации от энергии электронов, которая в свою очередь для каждого из потоков определяется прежде всего распределением потенциала по объему слоя и разбросом начальных энергий этих электронов. Рассмотрим особенности распределения потенциала по толщине слоя Ленгмюра, влияющие на распределение скоростей электронов. Прежде всего отметим, что основное падение потенциала происходит вблизи стенки. Типичное

Письма в ЖТФ, 2017, том 43, вып. 14 


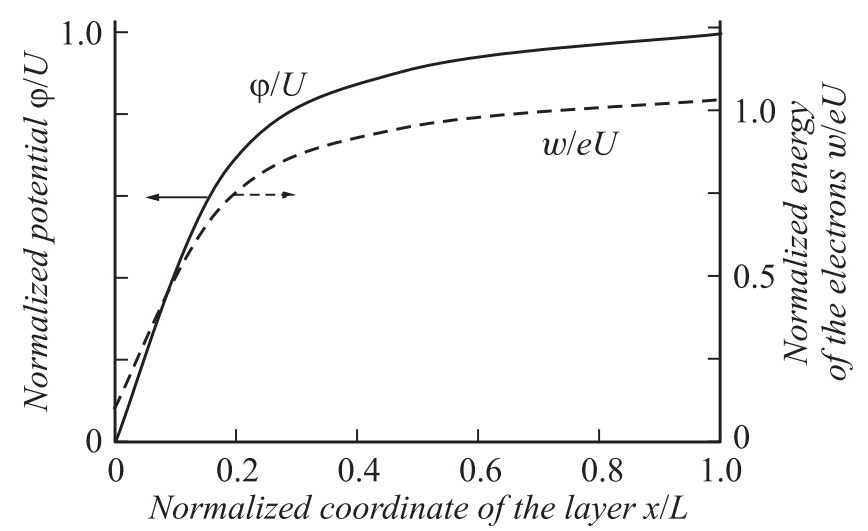

Рис. 2. Нормализованные распределения потенциала и энергии электронов по сечению слоя Ленгмюра. Пунктирная кривая относится как к энергии электронов, эмитированных из плазмы, так и к энергии электронов, эмитированных с поверхности материальной стенки. Стенка соответствует $x=0$, а $L-$ толщина слоя.

изменение потенциала показано на рис. 2. Учитывая, что вылетающие из плазмы электроны, энергия которых меньше $e U$, отражаются от слоя, получаем, что при $e U \gg k T_{e}$ основная часть электронов практически не проникает в слой и не влияет на эффекты, связанные с процессом ионизации атомов в слое. Таким образом, в первом приближении можно считать, что энергия электронов, эмитированных из плазмы, в зоне слабого изменения потенциала равна $e U$. Аналогичный вывод можно сделать и по отношению к электронам, эмитированным с поверхности стенки, поскольку они разгоняются вблизи стенки до энергии, близкой к $e U$, и в дальнейшем их энергия не меняется.

В предположении постоянства энергии электронов частота ионизации атомов, записывающаяся в виде $v_{i}=\sigma\left(j_{e}+j_{e m}\right) / e$, определяется плотностями тока электронов и в соответствии с (1) и (2) зависит от параметров пристеночной плазмы, температуры и работы выхода расплава, а также от параметров слоя Ленгмюра $U$ и $E$. При этом важно, что частота ионизации наиболее чувствительна к параметрам $U$ и $E$, поскольку последние определяют величины аргументов в экспоненциальных множителях плотностей токов.

Письма в ЖТФ, 2017, том 43, вып. 14 
Отметим, однако, что зависимость суммы плотностей токов от $U$ исчезает при использовании условия $j_{i}=j_{e}-j_{e m}$ [6], означающего равенство нулю суммарного тока в слое Ленгмюра. Здесь $j_{i}-$ плотность тока ионов, которая равна

$$
j_{i}=e n_{p l}\left(\frac{k T_{e}}{m_{i}}\right)^{1 / 2}
$$

где $m_{i}$ - масса иона. В этом случае частота ионизации атомов становится чувствительной только к $j$, т.е. к напряженности электрического поля $E$ на поверхности стенки

$$
v_{i}=\sigma\left(j_{i}+2 j_{e n}\right) / e .
$$

Для расчета напряженности этого электрического поля обычно используется классическая формула Маккоуна [7], которая с учетом условия $j_{i}=j_{e}-j_{e m}$ имеет вид

$$
E^{2} \approx \frac{2}{\varepsilon_{0}} \sqrt{\frac{m U}{e}}\left(j_{i}-2 \sqrt{\frac{m_{e}}{m_{i}}} j_{e m}\right) .
$$

$\mathrm{C}$ другой стороны, из уравнения Пуассона для распределения потенциала в слое $\Delta \varphi=-q / \varepsilon_{0}$, где $q-$ плотность заряда в слое, получаем, что напряженность электрического поля $E$ определяется полным зарядом слоя над единичной поверхностью стенки

$$
\varepsilon_{0} E=\int_{0}^{L} q d x,
$$

где $L-$ толщина слоя, которая зависит от температуры плазмы $T_{p l}$ и плотности плазмы на границе со слоем и составляет несколько длин Дебая $\lambda_{D}=\sqrt{\varepsilon_{0} k T_{p l} / e^{2} n_{p l}}$. Важно отметить, что соотношение (6) остается справедливым и при учете процесса ионизации атомов в слое, поскольку акт ионизации атомов не увеличивает полный заряд слоя. Соответственно мы можем использовать формулу (2) даже в случае, когда эффект ионизации атомов становится значительным.

Формула (5) совместно с условием баланса токов и при учете зависимостей (2) и (3) может рассматриваться как система уравнений 
для поиска двух неизвестных $U$ и $E$. Однако получающаяся при таком подходе система двух трансцендентных уравнений имеет лишь численное решение. Приближенное аналитическое решение может быть получено в случае пренебрежения вторым членом в правой части формулы (5), когда она приобретает существенно более простой вид $\varepsilon_{0} E^{2} \approx n_{p l} \sqrt{e U k T_{e}}$ и поправка Шоттки к работе выхода $\Delta w$, играющая ключевую роль при вычислении плотности тока эмиссии, записывается в виде

$$
\Delta w=\frac{e^{13 / 8}\left(U k T_{e} n_{p l}^{2}\right)^{1 / 8}}{2^{3 / 4} \sqrt{\pi} \varepsilon_{0}^{3 / 4}}
$$

Как видно из (7), зависимость $\Delta \omega$ от разности потенциалов $U$ является слабой, и в типичном интервале изменения разности потенциалов от 5 до $100 \mathrm{~V}$ зависимость $U^{1 / 8}$ с точностью около $15 \%$ можно заменить постоянной величиной $1.5 \mathrm{~V}^{1 / 8}$. Соответственно, ток эмиссии в этом случае определяется только параметрами приграничной плазмы и стенки

$$
j_{\text {em }}=A_{R} T^{2} \exp \left(-\frac{w}{k T}+\frac{e^{13 / 8}\left(1.5 k T_{e} n_{p l}^{2}\right)^{1 / 8}}{2^{3 / 4} \sqrt{\pi} \varepsilon_{0}^{3 / 4} k T}\right) .
$$

Коэффициент экранирования атомов слоем Ленгмюра равен доле атомов, ионизованных в этом слое. Исходя из уравнения непрерывности для атомов $v_{a} d n_{a} / d x=-v_{i}$, где $v_{a}$ и $n_{a}-$ скорость атомов и их плотность, этот коэффициент можно записать в виде

$$
K_{s c}=1=\exp \left(-v_{i} L / v_{a}\right) .
$$

Расчеты, проведенные с учетом того, что объединение формул (3), (4) и (8) позволяет вычислить частоту ионизации атомов, показали, что при $\sigma \approx 10^{-20} \mathrm{~m}^{2}, v_{a} \approx 300 \mathrm{~m} / \mathrm{s}, w=4.5 \mathrm{eV}, T=3000 \mathrm{~K}$ и $T_{e}=10 \mathrm{eV}$, которые типичны для большого числа экспериментов, коэффициент $K_{s c}$ становится близким к единице, когда плотность плазмы превосходит $8 \cdot 10^{19} \mathrm{~cm}^{3}$. Кроме того, сравнение плотности ионного тока с током эмиссии подтвердило правомерность пренебрежения током эмиссии в формуле (5) при плотности плазмы меньшей $3 \cdot 10^{20} \mathrm{~cm}^{3}$. Отметим, что этот диапазон плотностей пристеночной плазмы характерен для многих экспериментов по лазерной абляции металлов.

Письма в ЖТФ, 2017, том 43, вып. 14 
Таким образом, проведенные оценки позволили определить условия, при которых большая часть потока испаренных атомов экранируется слоем Ленгмюра. Однако следует отметить, что масса пристеночной плазмы и скорость эрозии материала стенки за счет испарения определяются не только долей потока испаренных атомов, достигшего границы плазмы, но и интенсивностью обратного потока ионов из плазмы. Соответственно близость коэффициента $K_{s c}$ к единице является необходимым, но не единственным условием для определения важности учета эффекта экранирования потока испаренных атомов; требуется также, чтобы поток испаренных атомов был сравним с потоком ионов из плазмы.

Оценим соотношение этих потоков для условий экспериментов по лазерной абляции металлов. Поток испаренных атомов равен $\Gamma_{a}=n_{w} D$, где $n_{w}$ - плотность расплава на поверхности стенки, а $D-$ скорость фронта плоской волны испарения, распространяющейся внутрь расплава. Как показано в [8], при лазерной абляции металлов эта скорость пропорциональна плотности потока энергии лазерного пучка $q$ равна $D \approx 5 \cdot 10^{-8} q$, где $[q]=\mathrm{GW} / \mathrm{cm}^{2}$. При проведении сравнения потока атомов с потоком ионов на стенку, определяемым по формуле (3), нами учтено, что в соответствии с результатами работы [9] плотность ионов на границе плазмы также пропорциональна параметру $q$ и может быть записана в виде $n_{p l} \approx q n_{w} / 20$. Из полученных формул следует, что потоки атомов и ионов сравнимы, если температура электронов вблизи стенки составляет несколько электрон-вольт, что типично для экспериментов по лазерной абляции металлов.

Таким образом, результаты исследований, проведенных в данной работе, указывают на необходимость учета эффекта экранирования потока испаренных атомов слоем Ленгмюра во многих случаях, в частности при моделировании процессов динамики лазерного факела.

В.М. Кожевин благодарит за поддержку Министерство образования и науки РФ, субсидия 14.613.21.0014, уникальный идентификатор проекта RFMEFI61314X0014.

\section{Список литературы}

[1] Hassanein A. // Probl. Atom. Sci. Technol. 2006. V. S6 (12). P. 130-134.

[2] Blank D.H.A., Dekkers M. et al. // J. Phys. D: Appl. Phys. 2014. V. 47. P. 034006.

Письма в ЖТФ, 2017, том 43, вып. 14 
[3] Рожанский B.A. Теория плазмы: Учебное пособие. СПб.: Лань, 2012. 320 с.

[4] Crowell C.R. // Solid-State Electron. 1965. V. 8 (4). P. 395-399.

[5] Orloff J. // Handbook of Charged Particle Optics. CRC Press, 2008.

[6] Bellis Isak L. // Laser Part. Beams. 2007. V. 25. P. 53-63.

[7] Mackeown S.S. // Phys. Rev. 1929. V. 34. P. 611-614.

[8] Анисимов С.И., Трибельский М.И., Эпельбаум Я.Г. // ЖЭТФ. 1980. Т. 78. В. 4. C. $1597-1605$.

[9] Анисимов С.И. // ЖЭТФ. 1968. Т. 54. С. 339-342.

Письма в ЖТФ, 2017, том 43, вып. 14 